\title{
An Efficient Attack on All Concrete KKS Proposals
}

\author{
Ayoub Otmani ${ }^{1,2}$ Jean-Pierre Tillich ${ }^{1}$ \\ 1 SECRET Project - INRIA Rocquencourt \\ Domaine de Voluceau, B.P. 10578153 Le Chesnay Cedex - France \\ ayoub.otmani@inria.fr, jean-pierre.tillich@inria.fr \\ 2 GREYC - Université de Caen - Ensicaen \\ Boulevard Maréchal Juin, 14050 Caen Cedex, France.
}

\begin{abstract}
Kabastianskii, Krouk and Smeets proposed in 1997 a digital signature scheme based on a couple of random error-correcting codes. A variation of this scheme was proposed recently and was proved to be EUF-1CMA secure in the random oracle model. In this paper we investigate the security of these schemes and suggest a simple attack based on (essentially) Stern's algorithm for finding low weight codewords. It efficiently recovers the private key of all schemes of this type existing in the literature. This is basically due to the fact that we can define a code from the available public data with unusual properties: it has many codewords whose support is concentrated in a rather small subset. In such a case, Stern's algorithm performs much better and we provide a theoretical analysis substantiating this claim. Our analysis actually shows that the insecurity of the proposed parameters is related to the fact that the rates of the couple of random codes used in the scheme were chosen to be too close. This does not compromise the security of the whole KKS scheme. It just points out that the region of weak parameters is really much larger than previously thought.
\end{abstract}

Keywords. Code-based cryptography, digital signature, random error-correcting codes, cryptanalysis.

\section{Introduction}

Digital signature schemes are probably among the most useful cryptographic algorithms. If quantum computers were to become reality, it would be useful to devise such schemes which would resist to it. A possible approach to meet this goal could be to build such schemes whose security relies on the difficulty of decoding linear codes. Two code based schemes of this kind have been proposed, namely the Courtois-Finiasz-Sendrier signature scheme [CFS01] and the Kabatianskii, Krouk and Smeets (KKS) scheme [KKS97,KKS05].

The Courtois-Finiasz-Sendrier (CFS) scheme presents the advantage of having an extremely short signature and its security has been proven to rely on the well-known syndrome decoding problem and the distinguishability of binary Goppa codes from a random code. However, it has been proved in $\left[\mathrm{FGO}^{+} 10\right]$ that the latter problem can be solved in the range of parameters used in the CFS signature algorithm. This does not prove that their proposal is insecure. However, it invalidates the hypotheses of their security proof. The main difficulty in suggesting a CFS type scheme is to come up with a family of very high rate codes with an efficient decoding algorithm and whose structure can be hidden in the same way as in the McEliece scheme. This narrows down quite a bit the families of codes which can be used in this setting and up to now only Goppa codes are known to meet this goal. It should be emphasized that it is precisely their rich algebraic structure which makes it possible to distinguish them from random codes.

On the other hand, the KKS proposal does not rely on Goppa codes and can be instantiated with random codes. Moreover, unlike in the CFS signature scheme, it does not compute a signature by using a decoding algorithm for the code and thus completely avoids the necessity of having to use restricted families of codes with a "hidden" trapdoor. Moreover, a variation of it has been proposed in [BMJ11] and has been proved to be EUF-1CMA secure in the random oracle model. The security of the KKS scheme has been investigated in [COV07]. It was shown that a passive attacker who may intercept just a few signatures can recover the private key. All the 
schemes proposed in [KKS97] can be broken in this way with the help of at most 20 signatures. The security of the scheme is not compromised by this attack however if only one signature is computed, and this especially in the variant proposed in [BMJ11] where some random noise is added on top of the signature.

The purpose of this article is to present a new security analysis of the KKS scheme and its variant proposed in [BMJ11]. Our approach for breaking the scheme is to define a certain error correcting code from the couple of public matrices used in the scheme and to notice that certain rather low weight codewords give actually valid signatures. It is therefore natural to use standard algorithms for finding low-weight codewords in this setting, such as Stern's algorithm [Ste88] or its Dumer variant [Dum96,FS09] (see also [BLP11]). It turns out that such algorithms are unusually successful in this setting due to the conjunction of three factors: (i) there are many low-weight codewords, (ii) they are localized on a rather small support, (iii) some part of this support is known to the attacker. It appears that all parameters suggested in [KKS97,KKS05,BMJ11] are easily broken by this approach and this without even knowing a single signature pair. Moreover, this approach can exploit the knowledge of a message-signature pair which speeds up the attack.

We provide an analysis of this attack which explains what makes it feasible for the parameters proposed in [KKS97,KKS05,BMJ11]. The KKS scheme relies on a couple of matrices which can be viewed as parity-check matrices of two linear codes. We show that when the first code has a rate which is smaller than the rate of the second one (or has approximately the same rate), then our attack is quite successful. This was exactly the case for all the parameters suggested in the past. In other words, our attack does not compromise the security of the whole KKS scheme. It just points out that the region of weak parameters is really much larger than previously thought.

\section{Terminology and Notation}

In the whole paper $q$ denotes some prime power and we denote by $\mathbb{F}_{q}$ the finite field with $q$ elements. Let $n$ be a non-negative integer. The set of integers $i$ such that $1 \leqslant i \leqslant n$ is denoted by $[1 \cdots n]$. The cardinality of a set $A$ is denoted by $|A|$. The concatenation of the vectors $\boldsymbol{x}=\left(x_{1}, \ldots, x_{n}\right)$ and $\boldsymbol{y}=\left(y_{1}, \ldots, y_{m}\right)$ is denoted by $(\boldsymbol{x} \| \boldsymbol{y}) \stackrel{\text { def }}{=}\left(x_{1}, \ldots, x_{n}, y_{1}, \ldots, y_{m}\right)$. The $\operatorname{support} \operatorname{supp}(\boldsymbol{x})$ of $\boldsymbol{x} \in \mathbb{F}_{q}^{n}$ is the set of $i$ 's such that $x_{i} \neq 0$. The (Hamming) weight $|\boldsymbol{x}|$ is the cardinality of $\operatorname{supp}(\boldsymbol{x})$. For a vector $\boldsymbol{x}=\left(x_{i}\right)$ and a subset $I$ of indices of $\boldsymbol{x}$, we denote by $\boldsymbol{x}_{I}$ its restriction to the indices of $I$, that is:

$$
\boldsymbol{x}_{I} \stackrel{\text { def }}{=}\left(x_{i}\right)_{i \in I} .
$$

We will also use this notation for matrices, in this case it stands for the submatrix formed by the columns in the index set, i.e. for any $k \times n$ matrix $\boldsymbol{H}$

$$
\boldsymbol{H}_{J} \stackrel{\text { def }}{=}\left(h_{i j}\right)_{\substack{1 \leqslant i \leqslant k \\ j \in J}}
$$

A linear code $\mathscr{C}$ of type $[n, k, d]$ over $\mathbb{F}_{q}$ is a linear subspace of $\mathbb{F}_{q}^{n}$ of dimension $k$ and minimum distance $d$ where by definition $d \stackrel{\text { def }}{=} \min \{|\boldsymbol{x}|: \boldsymbol{x} \in \mathscr{C}$ and $\boldsymbol{x} \neq \mathbf{0}\}$. The elements of $\mathscr{C}$ are codewords. A linear code can be defined either by a parity check matrix or a generator matrix. A parity check matrix $\boldsymbol{H}$ for $\mathscr{C}$ is an $(n-k) \times n$ matrix such that $\mathscr{C}$ is the right kernel of $\boldsymbol{H}$ :

$$
\mathscr{C}=\left\{\boldsymbol{c} \in \mathbb{F}_{q}^{n}: \boldsymbol{H} c^{T}=0\right\}
$$

where $\boldsymbol{x}^{T}$ denotes the transpose of $\boldsymbol{x}$. A generator matrix $\boldsymbol{G}$ is a $k \times n$ matrix formed by a basis of $\mathscr{C}$. We say that $\boldsymbol{G}$ is in systematic form if there exists a set $J$ such that $\boldsymbol{G}_{J}=\boldsymbol{I}_{k}$. The syndrome $\boldsymbol{s}$ by $\boldsymbol{H}$ of $\boldsymbol{x} \in \mathbb{F}_{q}^{n}$ is defined as $\boldsymbol{s}^{T} \stackrel{\text { def }}{=} \boldsymbol{H} \boldsymbol{x}^{T}$. A decoding algorithm for $\boldsymbol{H}$ is an algorithm such that, given $s$ in $\mathbb{F}_{q}^{r}$, finds a vector $\boldsymbol{e}$ of minimum weight whose syndrome is $s$. 


\section{The Kabatianskii-Krouk-Smeets Signature Scheme and its Variant}

This section is devoted to the description of two code-based signature schemes proposed in [KKS97] and more recently in [BMJ11], where the latter can be viewed as a "noisy" version of the former [KKS97]. Our presentation presents the main ideas without giving all the details which can be found in the original papers. We first focus on the scheme of [KKS97] whose construction relies on the following ingredients:

1. a full rank binary matrix $\boldsymbol{H}$ of size $(N-K) \times N$ with entries in a finite field $\mathbb{F}_{q}$.

2. a subset $J$ of $\{1, \ldots, N\}$ of cardinality $n$,

3. a linear code $\mathscr{C}_{\text {hidden }}$ over $\mathbb{F}_{q}$ of length $n \leqslant N$ and dimension $k$ defined by a generator matrix $\boldsymbol{G}$ of size $k \times n$. Let $t_{1}$ and $t_{2}$ be two integers such that with very high probability, we have that $t_{1} \leqslant|\boldsymbol{u}| \leqslant t_{2}$ for any non-zero codeword $\boldsymbol{u} \in \mathscr{C}_{\text {hidden }}$.

The matrix $\boldsymbol{H}$ is chosen such that the best decoding algorithms cannot solve the following search problem.

Problem 1. Given the knowledge of $s \in \mathbb{F}_{q}^{N-K}$ which is the syndrome by $\boldsymbol{H}$ of some $\boldsymbol{e} \in \mathbb{F}_{q}^{N}$ whose weight lies in $\left[t_{1} \cdots t_{2}\right]$, find explicitly $\boldsymbol{e}$, or eventually $\boldsymbol{x}$ in $\mathbb{F}_{q}^{N}$ different from $\boldsymbol{e}$ sharing the same properties as $\boldsymbol{e}$.

Finally let $\boldsymbol{F}$ be the $(N-K) \times k$ matrix defined by $\boldsymbol{F} \stackrel{\text { def }}{=} \boldsymbol{H}_{J} \boldsymbol{G}^{T}$. The Kabatianskii-Krouk-Smeets (KKS) signature scheme is then described in Figure 1.

Fig. 1. Description of the KKS scheme given in [KKS97].

- Setup.

1. The signer $S$ chooses $N, K n, k, t_{1}$ and $t_{2}$ according to the required security level.

2. $S$ draws a random $(N-K) \times N$ matrix $\boldsymbol{H}$.

3. $S$ randomly picks a subset $J$ of $\{1, \ldots, N\}$ of cardinality $n$.

4. $S$ randomly picks a random $k \times n$ generator matrix $G$ that defines a code $\mathscr{C}_{\text {hidden }}$ such that with high probability $t_{1} \leqslant|\boldsymbol{u}| \leqslant t_{2}$ for any non-zero codeword $\boldsymbol{u} \in \mathscr{C}_{\text {hidden }}$.

5. $\boldsymbol{F} \stackrel{\text { def }}{=} \boldsymbol{H}_{J} \boldsymbol{G}^{T}$ where $\boldsymbol{H}_{J}$ is the restriction of $\boldsymbol{H}$ to the columns in $J$.

- Keys.

- Private key. $J$ and $G$

- Public key. $\boldsymbol{F}$ and $\boldsymbol{H}$

- Signature. The signature $\sigma$ of a message $\boldsymbol{x} \in \mathbb{F}_{q}^{k}$ is defined as the unique vector $\sigma$ of $\mathbb{F}_{q}^{N}$ such that $\sigma_{i}=0$ for any $i \notin J$ and $\sigma_{J}=\boldsymbol{x} \boldsymbol{G}$.

- Verification. Given $(\boldsymbol{x}, \sigma) \in \mathbb{F}_{q}^{k} \times \mathbb{F}_{q}^{N}$, the verifier checks that $t_{1} \leqslant|\sigma| \leqslant t_{2}$ and $\boldsymbol{H} \sigma^{T}=\boldsymbol{F} \boldsymbol{x}^{T}$.

The scheme was modified in [BMJ11] to propose a one-time signature scheme by introducing two new ingredients, namely a hash function $f$ and adding an error vector $\boldsymbol{e}$ to the signature. It was proved that such a scheme is EUF-1CMA secure in the random oracle model. The description is given in Figure 2.

\section{Description of the Attack}

The purpose of this section is to explain the idea underlying our attack which aims at recovering the private key. The attack is divided in two main steps. First, we produce a valid signature for some message using only the public key. To do so, we build a new public code from matrices $\boldsymbol{H}$ and $\boldsymbol{F}$, and then we apply Dumer's algorithm [Dum91] in order to find low weight codewords that are closely related to codewords that belong to the hidden code $\mathscr{C}_{\text {hidden }}$ with high probability. Because 
Fig. 2. Description of the scheme of [BMJ11].

- Setup.

1. The signer $S$ chooses $N, K n, k, t_{1}$ and $t_{2}$ according to the required security level.

2. $S$ chooses a hash function $f:\{0,1\}^{*} \times \mathbb{F}_{2}^{N-K} \longrightarrow \mathbb{F}_{2}^{k}$.

3. $S$ draws a random binary $(N-K) \times N$ matrix $\boldsymbol{H}$.

4. $S$ randomly picks a subset $J$ of $\{1, \ldots, N\}$ of cardinality $n$.

5. $S$ randomly picks a $k \times n$ generator matrix $\boldsymbol{G}$ that defines a binary code $\mathscr{C}_{\text {hidden }}$ such that with high probability $t_{1} \leqslant|\boldsymbol{u}| \leqslant t_{2}$ for any non-zero codeword $\boldsymbol{u} \in \mathscr{C}_{\text {hidden }}$.

6. $\boldsymbol{F} \stackrel{\text { def }}{=} \boldsymbol{H}_{J} \boldsymbol{G}^{T}$ where $\boldsymbol{H}_{J}$ is the restriction of $\boldsymbol{H}$ to the columns in $J$.

- Keys.

- Private key. $J$ and $\boldsymbol{G}$

- Public key. $\boldsymbol{F}$ and $\boldsymbol{H}$

- Signature. The signature of a message $\boldsymbol{x} \in\{0,1\}^{*}$ is $(h, \sigma)$ defined as follows:

- $S$ picks a random $e \in \mathbb{F}_{2}^{N}$ such that $|\boldsymbol{e}|=n$.

- Let $\boldsymbol{h} \stackrel{\text { def }}{=} f\left(\boldsymbol{x}, \boldsymbol{H} \boldsymbol{e}^{T}\right)$ and $\boldsymbol{y}$ be the unique vector of $\mathbb{F}_{2}^{N}$ such that (i) $\operatorname{supp}(\boldsymbol{y}) \subset J$, (ii) $\boldsymbol{y}_{J}=\boldsymbol{h} \boldsymbol{G}$. The second part of the signature $\sigma$ is then given by $\sigma \stackrel{\text { def }}{=} \boldsymbol{y}+\boldsymbol{e}$.

- Verification. Given a signature $(\boldsymbol{h}, \sigma) \in \mathbb{F}_{2}^{k} \times \mathbb{F}_{2}^{N}$ for $\boldsymbol{x} \in\{0,1\}^{*}$, the verifier checks that $|\sigma| \leqslant 2 n$ and $\boldsymbol{h}=f\left(\boldsymbol{x}, \boldsymbol{H} \sigma^{T}+\boldsymbol{F} \boldsymbol{h}^{T}\right)$.

of this close relationship, we are able to produce one valid message/signature pair, and since each signature reveals partial information about the private key, we can reuse it to get another valid message/signature pair revealing more information. We repeat this process a few times until we totally recover the whole private key. More details will be given in the following sections.

In what follows, we make the assumption that all the codes are binary because all the concrete proposals are of this kind. The non-binary case will be discussed in the conclusion.

\subsection{An auxiliary code}

We give here the first ingredient we use to forge a valid message/signature pair for the KKS scheme just from the knowledge of the public pair $\boldsymbol{H}, \boldsymbol{F}$. This attack can also be used for the second scheme given by Figure 2 . In the last case, it is not a valid message/signature pair anymore but an auxiliary quantity which helps in revealing $J$. This ingredient consists in a linear code $\mathscr{C}_{\text {pub }}$ of length $N+k$ defined as the kernel of $\hat{\boldsymbol{H}}$ which is obtained by the juxtaposition of the two public matrices $\boldsymbol{H}$ and $\boldsymbol{F}$ as given in Figure 3. The reason behind this definition lies in the following Fact 1 .

Fig. 3. Parity-check matrix $\hat{\boldsymbol{H}}$ of the code $\mathscr{C}_{\text {pub }}$

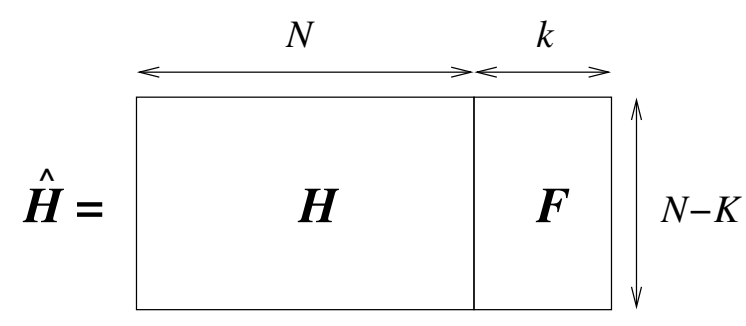


Fact 1. Let $\boldsymbol{x}^{\prime}$ be in $\mathbb{F}_{2}^{N+k}$ and set $(\sigma \| \boldsymbol{x}) \stackrel{\text { def }}{=} \boldsymbol{x}^{\prime}$ with $\sigma$ in $\mathbb{F}_{2}^{N}$ and $\boldsymbol{x}$ in $\mathbb{F}_{2}^{k}$. Then $\sigma$ is a signature of $\boldsymbol{x}$ if and only if:

1. $\hat{\boldsymbol{H}} \boldsymbol{x}^{T}=0$

2. $t_{1} \leqslant|\sigma| \leqslant t_{2}$.

The code $\mathscr{C}_{\text {pub }}$ is of dimension $k+K$, and of particular interest is the linear space $\mathscr{C}_{\text {sec }} \subset \mathscr{C}_{\text {pub }}$ that consists in words that satisfy both conditions of Fact 1 and that are obtained by all pairs $(\sigma, \boldsymbol{x})$ of valid message/signature, that is to say:

$$
\mathscr{C}_{\mathrm{sec}} \stackrel{\text { def }}{=}\left\{(\sigma \| \boldsymbol{x}) \in \mathbb{F}_{2}^{N+k}: \boldsymbol{x} \in \mathbb{F}_{2}^{k}, \sigma \in \mathbb{F}_{2}^{N}, \sigma_{J}=\boldsymbol{x} \boldsymbol{G}, \sigma_{[1 \cdots N] \backslash J}=0\right\} .
$$

Clearly, the dimension of $\mathscr{C}_{\sec }$ is $k$. Additionally, we expect that the weight of $\sigma$ is of order $n / 2$ for any $(\sigma, \boldsymbol{x})$ in $\mathscr{C}_{\text {sec }}$, which is much smaller than the total length $N$. This strongly suggests to use well-known algorithms for finding low weight codewords to reveal codewords in $\mathscr{C}_{\text {sec }}$ and therefore message/signature pairs. The algorithm we used for that purpose is specified in the following subsection.

\subsection{Finding low-weight codewords}

We propose to use the following variation on Stern's algorithm due to [Dum91] (See also [FS09]). The description of the algorithm is given in Algorithm 1. It consists in searching for low-weight codewords among the candidates that are of very low-weight $2 p$ (where $p$ is typically in the range $1 \leqslant p \leqslant 4)$ when restricted to a set $I$ of size slightly larger than the dimension $k+K$ of the code $\mathscr{C}_{\text {pub }}$, say $|I|=k+K+l$ for some small integer $l$. The key point in this approach is to choose $I$ among a set $S$ of test positions. The set $S$ will be appropriately chosen according to the considered context. If no signature pair is known, then a good choice for $S$ is to take:

$$
S=[1 \cdots N]
$$

This means that we always choose the test positions among the $N$ first positions of the code $\mathscr{C}_{\text {pub }}$ and never among the $k$ last positions. The reason for this choice will be explained in the following subsection.

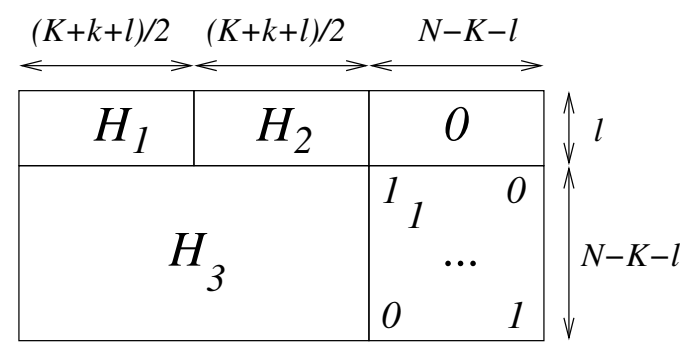

Fig. 4. A parity-check matrix for $\mathscr{C}_{\text {pub }}$ in quasi-systematic form.

\subsection{Explaining the success of the attack}

It turns out that this attack works extremely well on all the parameter choices made in the literature, and this even without knowing a single message/signature pair which would make life much easier for the attacker as demonstrated in [COV07]. In a first pass, the attack recovers easily message/signature pairs for all the parameters suggested in [BMJ11,KKS97,KKS05]. Once 


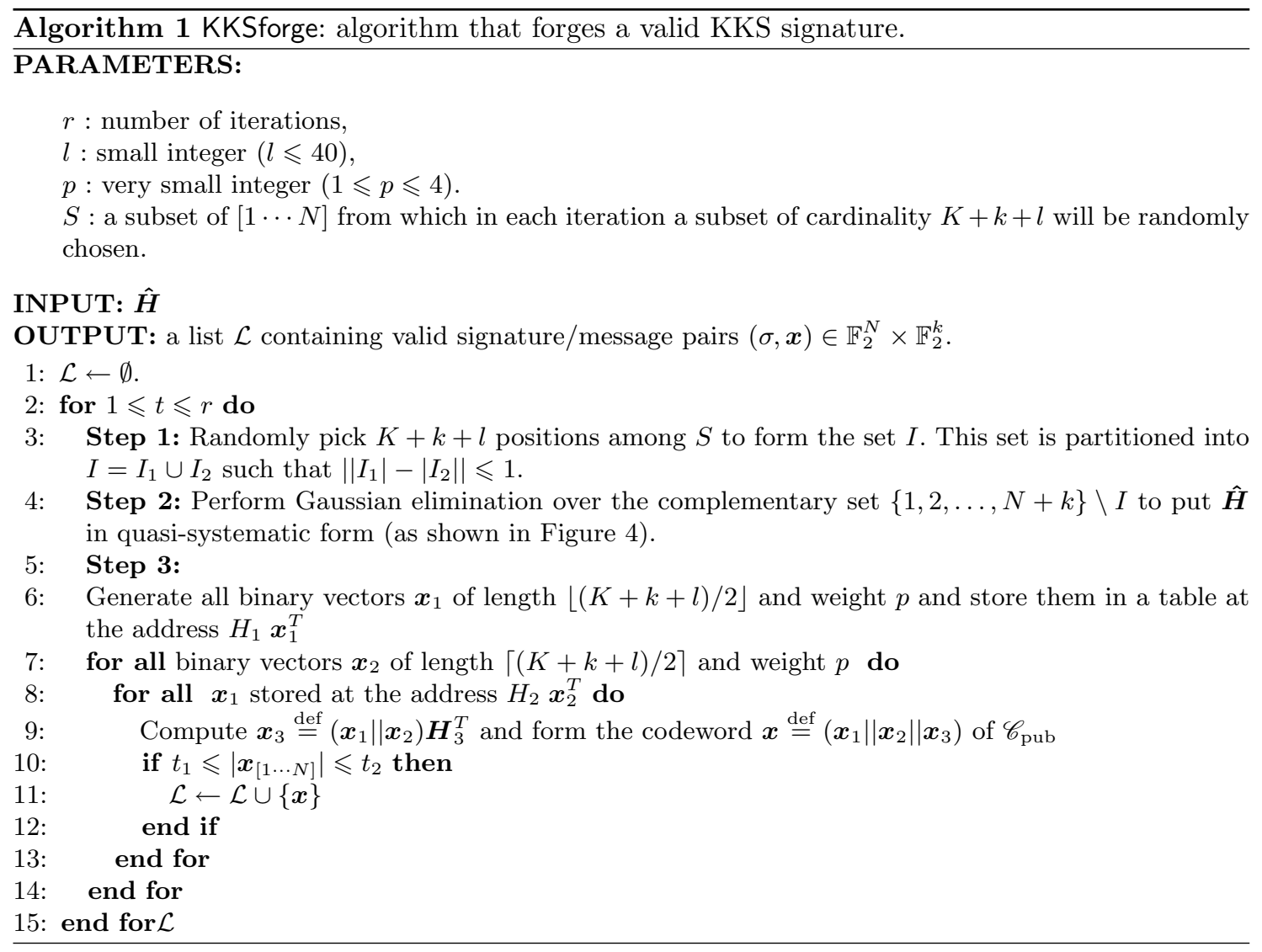

a signature/message pair is obtained, it can be exploited to bootstrap an attack that recovers the private key as we will explain later.

The reason why the attack works much better here than for general linear codes comes from the fact that $\hat{\boldsymbol{H}}$ does not behave like a random matrix at all even if the two chosen matrices for the scheme, namely $\boldsymbol{H}$ and $\boldsymbol{G}$ are chosen at random. The left part and the right part $\boldsymbol{H}$ and $\boldsymbol{F}$ are namely related by the equation:

$$
\boldsymbol{F}=\boldsymbol{H}_{J} \boldsymbol{G}^{T} .
$$

Indeed, the parity-check matrix $\hat{\boldsymbol{H}}$ displays peculiar properties: $\mathscr{C}_{\text {pub }}$ contains $\mathscr{C}_{\text {sec }}$ as a subcode and its codewords are precisely what we would like to find in order to generate valid message/signature pairs. This subcode has actually a very specific structure that helps greatly the attacker:

1. There are many codewords in $\mathscr{C}_{\text {sec }}$, namely $2^{k}$.

2. The support of these codewords is included in a fixed (and rather small) set of size $k+n$.

3. $k$ positions of this set are known to the attacker.

4. These codewords form a linear code (of dimension $k$ ).

Because of all these properties, the aforementioned attack will work much better than should be expected from a random code. More precisely, let us bring in:

$$
I^{\prime} \stackrel{\text { def }}{=} I \cap J
$$

Notice that the expectation $\mathbb{E}\left\{\left|I^{\prime}\right|\right\}$ of the cardinality of the set $I^{\prime}$ is equal to:

$$
\mathbb{E}\left\{\left|I^{\prime}\right|\right\}=\frac{n}{N}(k+K+l)=(R+\alpha \rho+\lambda) n
$$


where we introduced the following notation:

$$
R \stackrel{\text { def }}{=} \frac{K}{N}, \quad \rho \stackrel{\text { def }}{=} \frac{k}{n}, \quad \alpha \stackrel{\text { def }}{=} \frac{n}{N} \quad \text { and } \quad \lambda \stackrel{\text { def }}{=} \frac{l}{N} .
$$

The point is that whenever there is a codeword $\boldsymbol{c}$ in $\mathscr{C}_{\mathrm{sec}}$ which is such that $\left|\boldsymbol{c}_{I^{\prime}}\right|=2 p$ we have a non-negligible chance to find it with Algorithm 1. This does not hold with certainty because the algorithm does not examine all codewords $\boldsymbol{x}$ such that $\left|\boldsymbol{x}_{I}\right|=2 p$, but rather it consists in splitting $I$ in $I_{1}$ and $I_{2}$ of the same size and looking for codewords $\boldsymbol{x}$ such that $\left|\boldsymbol{x}_{I_{1}}\right|=\left|\boldsymbol{x}_{I_{2}}\right|=p$. In other words, we consider only a fraction $\delta$ of such codewords where:

$$
\delta=\frac{\left(\begin{array}{c}
(K+k+l) / 2 \\
p
\end{array}\right)\left(\begin{array}{c}
(K+k+l) / 2 \\
p
\end{array}\right)}{\left(\begin{array}{c}
K+k+l \\
2 p
\end{array}\right)} \approx \sqrt{\frac{(K+k+l)}{\pi p(K+k+l-2 p)}} .
$$

We will therefore obtain all codewords $\boldsymbol{c}$ in $\mathscr{C}_{\text {sec }}$ which are such that $\left|\boldsymbol{c}_{I_{1}}\right|=\left|\boldsymbol{c}_{I_{2}}\right|=p$. Consider now the restriction $\mathscr{C}_{\mathrm{sec}}^{\prime}$ of $\mathscr{C}_{\mathrm{sec}}$ to the positions belonging to $I^{\prime}$, that is:

$$
\mathscr{C}_{\mathrm{sec}}^{\prime}=\left\{\left(\boldsymbol{x}_{i}\right)_{i \in I^{\prime}}: \boldsymbol{x}=\left(\boldsymbol{x}_{i}\right)_{i \in[1 \cdots N+k]} \in \mathscr{C}_{\mathrm{sec}}\right\}
$$

The crucial issue is now the following question:

$$
\text { Does there exist in } \mathscr{C}_{\text {sec }}^{\prime} \text { a codeword of weight } 2 p \text { ? }
$$

The reason for this is explained by the following proposition.

Proposition 1. Let $I_{s}^{\prime} \stackrel{\text { def }}{=} I_{s} \cap J$ for $s \in\{1,2\}$. If there exists a codeword $\boldsymbol{x}^{\prime}$ in $\mathscr{C}_{\text {sec }}^{\prime}$ such that $\left|\boldsymbol{x}_{I_{1}^{\prime}}^{\prime}\right|=\left|\boldsymbol{x}_{I_{2}^{\prime}}^{\prime}\right|=p$, then it will be the restriction of a codeword $\boldsymbol{x}$ in $\mathscr{C}_{\text {sec }}$ which will belong to the list $\mathcal{L}$ output by Algorithm 1.

Proof. Consider a codeword $\boldsymbol{x}^{\prime}$ in $\mathscr{C}_{\mathrm{sec}}^{\prime}$ such that $\left|\boldsymbol{x}_{I_{1}^{\prime}}^{\prime}\right|=\left|\boldsymbol{x}_{I_{2}^{\prime}}^{\prime}\right|=p$. For $s \in\{1,2\}$, extend $\boldsymbol{x}_{I_{s}^{\prime}}$ with zeros on the other positions of $I_{s}$ and let $\boldsymbol{x}_{s}$ be the corresponding word. Notice that $\boldsymbol{x}_{1}$ and $\boldsymbol{x}_{2}$ will be considered by Algorithm 1 and $\boldsymbol{x}_{1}$ will be stored at the address $\boldsymbol{H}_{1} \boldsymbol{x}_{1}^{T}$. By definition of $\boldsymbol{x}^{\prime}$, $\left(\boldsymbol{x}_{1} \| \boldsymbol{x}_{2}\right)$ is the restriction of a codeword $\boldsymbol{x}$ of $\mathscr{C}_{\text {sec }}$ to $I$, say $\boldsymbol{x}=\left(\boldsymbol{x}_{1}\left\|\boldsymbol{x}_{2}\right\| \boldsymbol{y}\right)$ with $\boldsymbol{y} \in \mathbb{F}_{2}^{N-K-l}$. Since $\mathscr{C}_{\text {sec }} \subset \mathscr{C}_{\text {pub }}$ we have $\hat{\boldsymbol{H}} \boldsymbol{x}^{T}=0$. Let $\hat{\boldsymbol{H}}^{\prime}$ be the matrix obtained from $\hat{\boldsymbol{H}}$ put in quasi-systematic form through a Gaussian elimination as given in Figure 4. We also have $\hat{\boldsymbol{H}}^{\prime} \boldsymbol{x}^{T}=0$ and hence:

$$
\boldsymbol{H}_{1} \boldsymbol{x}_{1}^{T}+\boldsymbol{H}_{2} \boldsymbol{x}_{2}^{T}=0
$$

and

$$
\boldsymbol{H}_{3}\left(\boldsymbol{x}_{1} \| \boldsymbol{x}_{2}\right)^{T}+\boldsymbol{y}^{T}=0 .
$$

Equation (5) shows that $\boldsymbol{x}_{1}$ is stored at address $\boldsymbol{H}_{2} \boldsymbol{x}_{2}^{T}$ and will be considered at Step 4.2 of the algorithm. In this case, $\boldsymbol{x}$ will be stored in $\mathcal{L}$.

We expect that the dimension of $\mathscr{C}_{\mathrm{sec}}^{\prime}$ is still $k$ and that this code behaves like a random code of the same length and dimension. Ignoring the unessential issue whether or not $\boldsymbol{x}^{\prime}$ satisfies $\left|\boldsymbol{x}_{I_{1}^{\prime}}^{\prime}\right|=\left|\boldsymbol{x}_{I_{2}^{\prime}}^{\prime}\right|=p$, let us just assume that there exists $\boldsymbol{x}^{\prime}$ in $\mathscr{C}_{\text {sec }}^{\prime}$ such that $\left|\boldsymbol{x}^{\prime}\right|=2 p$. There is a non negligible chance that we have $\left|\boldsymbol{x}_{I_{1}^{\prime}}^{\prime}\right|=\left|\boldsymbol{x}_{I_{2}^{\prime}}^{\prime}\right|=p$ and that this codeword will be found by our algorithm. The issue is therefore whether or not there is a codeword of weight $2 p$ in a random code of dimension $k$ and length $\left|I^{\prime}\right|$. This holds with a good chance (see [BF02] for instance) as soon as:

$$
2 p \geqslant d_{\mathrm{GV}}\left(\left|I^{\prime}\right|, k\right)
$$

where $d_{\mathrm{GV}}\left(\left|I^{\prime}\right|, k\right)$ denotes the Gilbert-Varshamov distance of a code of length $\left|I^{\prime}\right|$ and dimension $k$. Recall that [MS86]:

$$
d_{\mathrm{GV}}\left(\left|I^{\prime}\right|, k\right) \approx h^{-1}\left(1-k /\left|I^{\prime}\right|\right)\left|I^{\prime}\right|
$$


where $h^{-1}(x)$ is the inverse function defined over $\left[0, \frac{1}{2}\right]$ of the binary entropy function $h(x) \stackrel{\text { def }}{=}$ $-x \log _{2} x-(1-x) \log _{2}(1-x)$. Recall that we expect to have:

$$
\left|I^{\prime}\right| \approx(R+\alpha \rho+\lambda) n
$$

which implies

$$
\frac{k}{\left|I^{\prime}\right|} \approx \frac{\rho}{R+\alpha \rho+\lambda} \approx \frac{\rho}{R}
$$

when $\alpha$ and $\lambda$ are small. Roughly speaking, to avoid such an attack, several conditions have to be met:

1. $\rho$ has to be significantly smaller than $R$,

2. $n$ has to be large enough.

This phenomenon was clearly not taken into in the parameters suggested in [KKS97,KKS05,BMJ11] as shown in Table 1 . The values of $d_{\mathrm{GV}}\left(\left|I^{\prime}\right|, k\right)$ are extremely low (in the range $1-6$ ). In other words, taking $p=1$ is already quite threatening for all these schemes. For the first parameter set, namely $(k, n, K, N)=(60,1023,192,3000)$, this suggests to take $p=3$. Actually taking $p=1$ gives an attack with less complexity. More iterations have to be performed but each iteration is less complex.

Finally, let us observe that when this attack gives a message/signature pair, it can be used as a bootstrap for an attack that recovers the whole private key as will be explained in the following subsection.

Table 1. KKS Parameters with the corresponding value of $d_{\mathrm{GV}}\left(n^{\prime}, k\right)$.

\begin{tabular}{l|c|r|c|c|c|r|r} 
Article & $\rho$ & $n$ & $l$ & $n^{\prime} \stackrel{\text { def }}{=} \mathbb{E}\left\{\left|I^{\prime}\right|\right\}$ & $R$ & $N$ & $d_{\mathrm{GV}}\left(n^{\prime}, k\right)$ \\
\hline$[$ KKS97] & $\frac{60}{1023} \approx 0.059$ & 1,023 & 8 & 89 & $\frac{192}{3000} \approx 0.064$ & 3,000 & 6 \\
\hline$[$ KKS05] & $\frac{48}{255} \approx 0.188$ & 255 & 8 & 65 & $\frac{260}{1200} \approx 0.208$ & 1,200 & 3 \\
\hline$[$ KKS97] & $\frac{48}{180} \approx 0.267$ & 180 & 8 & 64 & $\frac{335}{1100} \approx 0.305$ & 1,100 & 3 \\
\hline$[$ BMJ11] & $1 / 2$ & 320 & 12 & 165 & $1 / 2$ & 11,626 & 1 \\
\hline$[$ BMJ11] & $1 / 2$ & 448 & 13 & 230 & $1 / 2$ & 16,294 & 1 \\
\hline$[$ BMJ11] & $1 / 2$ & 512 & 13 & 264 & $1 / 2$ & 18,586 & 1 \\
\hline$[$ BMJ11] & $1 / 2$ & 768 & 13 & 395 & $1 / 2$ & 27,994 & 2 \\
\hline$[$ BMJ11] & $1 / 2$ & 1,024 & 14 & 527 & $1 / 2$ & 37,274 & 2
\end{tabular}

\subsection{Exploiting a signature for extracting the private key}

If a signature $\sigma$ of a message $\boldsymbol{x}$ is known, then $\boldsymbol{y} \stackrel{\text { def }}{=}(\sigma, \boldsymbol{x})$ is a codeword of $\mathscr{C}_{\text {sec }}$ which has weight about $n / 2$ when restricted to its $N$ first positions. This yields almost half of the positions of $J$. This can be exploited as follows. We perform the same attack as in the previous subsection, but we avoid choosing positions $i$ for which $\sigma_{i}=1$. More precisely, if we let $J_{\sigma} \stackrel{\text { def }}{=} \operatorname{supp}(\sigma)=\left\{i: \sigma_{i}=1\right\}$, then we choose $K+k+l$ positions among $[1 \cdots N] \backslash J_{\sigma}$ to form $I$. The point of this choice is that we have more chances to have a smaller size for $I^{\prime}=I \cap J$. Let $n^{\prime} \stackrel{\text { def }}{=}\left|I^{\prime}\right|$, we have now:

$$
\begin{gathered}
\mathbb{E}\left\{n^{\prime} \mid J_{\sigma}\right\}=\frac{n-\left|J_{\sigma}\right|}{N-\left|J_{\sigma}\right|}(k+K+l) \\
\mathbb{E}\left\{\left|I^{\prime}\right|\right\}=\mathbb{E}\left\{\mathbb{E}\left\{n^{\prime} \mid J_{\sigma}\right\}\right\} \approx \frac{n / 2}{(N-n / 2)}(k+K+l) .
\end{gathered}
$$


The last approximation follows from the fact that the weight $|\sigma|$ is quite concentrated around $n / 2$. The same reasoning can be made as before, but the odds that the algorithm finds other valid signatures are much higher. This comes from the fact that the expectation $\left|I^{\prime}\right|$ is half the expected size of $I^{\prime}$ in the previous case as given in Equation (3). Previously we had $\mathbb{E}\left\{\frac{\left|I^{\prime}\right|}{k}\right\} \approx \frac{R}{\rho}$, whereas now we have:

$$
\mathbb{E}\left\{\frac{\left|I^{\prime}\right|}{k}\right\} \approx \frac{R}{2 \rho} .
$$

In other words, in order to avoid the previous attack we had to take $\rho$ significantly smaller than $R$ and now, we have to take $\rho$ significantly smaller than $R / 2$. For all the parameters proposed in the past, it turns out that $d_{\mathrm{GV}}\left(\left|I^{\prime}\right|, k\right)$ is almost always equal to 1 , which makes the attack generally successful in just one iteration by choosing $p=1$.

Moreover, if another valid signature $\sigma^{\prime}$ is obtained and by taking the union $J_{\sigma} \cup J_{\sigma^{\prime}}$ of the supports, then about $3 / 4$ of the positions of $J$ will be revealed. We can start again the process of finding other message/signature pairs by choosing $K+k+l$ positions among $\{1,2, \ldots, N\} \backslash$ $\left(J_{\sigma} \cup J_{\sigma^{\prime}}\right)$ to form the sets $I$. This approach can be iterated as explained in Algorithm 2. This process will quickly reveal the whole set $J$ and from this, the private key is easily extracted as detailed in [COV07].

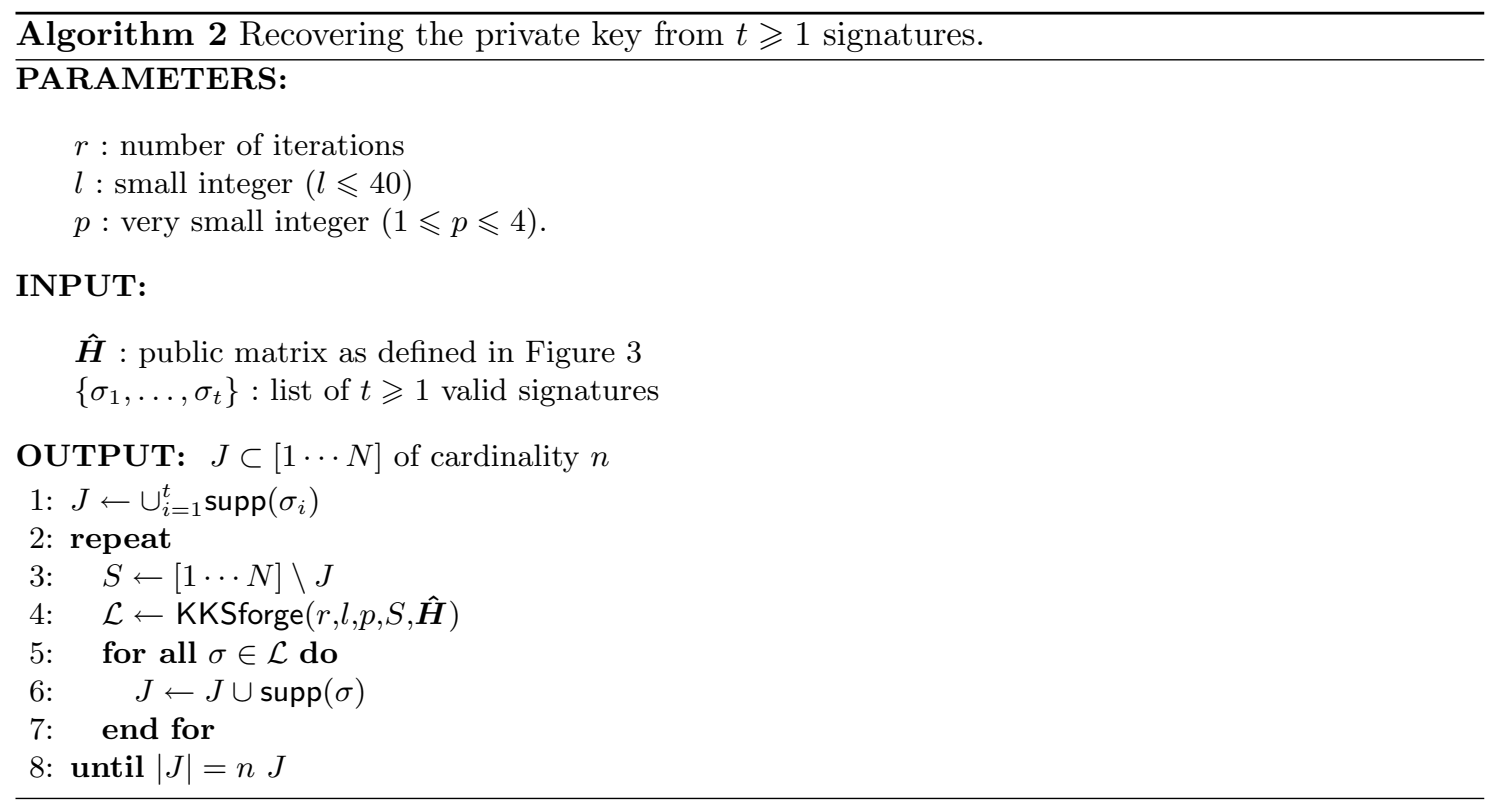

Finally, let us focus on the variant proposed in [BMJ11]. In this case, we have slightly less information than in the original KKS scheme. This can be explained by the following reasoning. In this case too, we choose $S$ again as $[1 \cdots N] \backslash J_{\sigma}$, where as before $J_{\sigma}$ is defined as $J_{\sigma} \stackrel{\text { def }}{=}\left\{i: \sigma_{i}=1\right\}$. However this time, by defining $n^{\prime}$ again as $n^{\prime} \stackrel{\text { def }}{=}\left|I^{\prime}\right|$, we have

$$
\mathbb{E}\left\{n^{\prime} \mid J_{\sigma}\right\}=\frac{\left|J_{\sigma}^{\prime}\right|}{N-\left|J_{\sigma}\right|}(k+K+l)
$$

where

$$
J_{\sigma}^{\prime}=J \backslash J_{\sigma} .
$$

However, this time due to the noise which is added, $\left|J_{\sigma}\right|$ is expected to be larger than before (namely of order $\frac{n}{2}+\frac{(N-n) n}{N}$ ). 


\section{Analysis of the Attack}

The purpose of this section is to provide a very crude upper-bound on the complexity of the attack. We assume here that the code $\mathscr{C}_{\text {rand }}$ of length $n$ which is equal to the restriction on $J$ of $\mathscr{C}_{\text {sec }}$ :

$$
\mathscr{C}_{\text {rand }} \stackrel{\text { def }}{=}\left\{\left(x_{j}\right)_{j \in J}: \boldsymbol{x}=\left(x_{1}, \ldots, x_{N+k}\right) \in \mathscr{C}_{\text {sec }}\right\}
$$

behaves as a random code. More precisely we assume that it has been chosen by picking a random parity-check matrix $\boldsymbol{H}_{\text {rand }}$ of size $(n-k) \times n$ (by choosing its entries uniformly at random among $\left.\mathbb{F}_{2}\right)$. This specifies a code $\mathscr{C}_{\text {rand }}$ of length $n$ as $\mathscr{C}_{\text {rand }}=\left\{\boldsymbol{x} \in \mathbb{F}_{2}^{n}: \boldsymbol{H}_{\text {rand }} \boldsymbol{x}^{T}=0\right\}$. We first give in the following section some quite helpful lemmas about codes of this kind.

\subsection{Preliminaries about random codes}

We are interested in this section in obtaining a lower bound on the probability that a certain subset $X$ of $\mathbb{F}_{2}^{n}$ has a non empty intersection with $\mathscr{C}_{\text {rand }}$. For this purpose, we first calculate the two following probabilities.

Lemma 1. Let $\boldsymbol{x}$ and $\boldsymbol{y}$ be two different and nonzero elements of $\mathbb{F}_{2}^{n}$. Then

$$
\begin{aligned}
\operatorname{prob}\left(\boldsymbol{x} \in \mathscr{C}_{\text {rand }}\right) & =2^{k-n} \\
\operatorname{prob}\left(\boldsymbol{x} \in \mathscr{C}_{\text {rand }}, \boldsymbol{y} \in \mathscr{C}_{\text {rand }}\right) & =2^{2(k-n)}
\end{aligned}
$$

To prove this lemma, we will introduce the following notation and lemma. For $\boldsymbol{x}=\left(x_{i}\right)_{1 \leqslant i \leqslant s}$ and $\boldsymbol{y}=\left(y_{i}\right)_{1 \leqslant i \leqslant s}$ being two elements of $\mathbb{F}_{2}^{s}$ for some arbitrary $s$, we define $\boldsymbol{x} \cdot \boldsymbol{y}$ as

$$
\boldsymbol{x} \cdot \boldsymbol{y}=\sum_{1 \leqslant i \leqslant s} x_{i} y_{i}
$$

the addition being performed over $\mathbb{F}_{2}$.

Lemma 2. Let $\boldsymbol{x}$ and $\boldsymbol{y}$ be two different and nonzero elements of $\mathbb{F}_{2}^{n}$ and choose $\boldsymbol{h}$ uniformly at random in $\mathbb{F}_{2}^{n}$, then

$$
\begin{array}{r}
\operatorname{prob}(\boldsymbol{x} \cdot \boldsymbol{h}=0)=\frac{1}{2} \\
\operatorname{prob}(\boldsymbol{x} \cdot \boldsymbol{h}=0, \boldsymbol{y} \cdot \boldsymbol{h}=0)=\frac{1}{4}
\end{array}
$$

Proof. To prove Equation (12) we just notice that the subspace $\left\{\boldsymbol{h} \in \mathbb{F}_{2}^{n}: \boldsymbol{x} \cdot \boldsymbol{h}=0\right\}$ is of dimension $n-1$. There are therefore $2^{n-1}$ solutions to this equation and

$$
\operatorname{prob}(\boldsymbol{x} \cdot \boldsymbol{h}=0)=\frac{2^{n-1}}{2^{n}}=\frac{1}{2} .
$$

On the other hand, the hypothesis made on $\boldsymbol{x}$ and $\boldsymbol{y}$ implies that $\boldsymbol{x}$ and $\boldsymbol{y}$ generate a subspace of dimension 2 in $\mathbb{F}_{2}^{n}$ and that the dual space, that is $\left\{\boldsymbol{h} \in \mathbb{F}_{2}^{n}: \boldsymbol{x} \cdot \boldsymbol{h}=0, \boldsymbol{y} \cdot \boldsymbol{h}=0\right\}$ is of dimension $n-2$. Therefore

$$
\operatorname{prob}(\boldsymbol{x} \cdot \boldsymbol{h}=0, \boldsymbol{y} \cdot \boldsymbol{h}=0)=\frac{2^{n-2}}{2^{n}}=\frac{1}{4}
$$

Proof (of Lemma 1). Let $\boldsymbol{h}_{1}, \ldots, \boldsymbol{h}_{n-k}$ be the $n-k$ rows of $\boldsymbol{H}_{\text {rand }}$. Then

$$
\begin{aligned}
\operatorname{prob}\left(\boldsymbol{x} \in \mathscr{C}_{\text {rand }}\right) & =\operatorname{prob}\left(\boldsymbol{H}_{\text {rand }} \boldsymbol{x}^{T}=0\right) \\
& =\operatorname{prob}\left(\boldsymbol{h}_{1} \cdot \boldsymbol{x}=0, \ldots, \boldsymbol{h}_{n-k} \cdot \boldsymbol{x}=0\right) \\
& =\operatorname{prob}\left(\boldsymbol{h}_{1} \cdot \boldsymbol{x}=0\right) \ldots \operatorname{prob}\left(\boldsymbol{h}_{n-k} \cdot \boldsymbol{x}=0\right) \\
& =2^{k-n}
\end{aligned}
$$

where Equation (14) follows by the independence of the events and Equation (15) uses Lemma 2. Equation (11) is obtained in a similar fashion. 
Lemma 3. Let $X$ be some subset of $\mathbb{F}_{2}^{n}$ of size $m$ and let $f$ be the function defined by $f(x) \stackrel{\text { def }}{=}$ $\max \left(x(1-x / 2), 1-\frac{1}{x}\right)$. We denote by $x$ the quantity $\frac{m}{2^{n-k}}$, then

$$
\operatorname{prob}\left(X \cap \mathscr{C}_{\text {rand }} \neq \emptyset\right) \geq f(x) .
$$

Proof. For $\boldsymbol{x}$ in $X$ we define $E_{\boldsymbol{x}}$ as the event " $\boldsymbol{x}$ belongs to $\mathscr{C}_{\text {rand }}$ " and we let $q \stackrel{\text { def }}{=} 2^{k-n}$. We first notice that

$$
\operatorname{prob}\left(X \cap \mathscr{C}_{\text {rand }} \neq \emptyset\right)=\operatorname{prob}\left(\bigcup_{x \in X} E_{x}\right) .
$$

By using the Bonferroni inequality [Com74, p. 193] on the probability of the union of events we obtain

$$
\begin{aligned}
\operatorname{prob}\left(\bigcup_{\boldsymbol{x} \in X} E_{\boldsymbol{x}}\right) & \geq \sum_{\boldsymbol{x} \in X} \operatorname{prob}\left(E_{\boldsymbol{x}}\right)-\sum_{\{\boldsymbol{x}, \boldsymbol{y}\} \subset X} \operatorname{prob}\left(E_{\boldsymbol{x}} \cap E_{\boldsymbol{y}}\right) \\
& \geq m q-\frac{m(m-1)}{2} q^{2} \\
& \geq m q-\frac{m^{2} q^{2}}{2} \\
& \geq m q(1-m q / 2)
\end{aligned}
$$

where (17) follows from Lemma 1. This bound is rather sharp for small values of $m q$. On the other hand for larger values of $m q$, another lower bound on $\operatorname{prob}\left(X \cap \mathscr{C}_{\text {rand }} \neq \emptyset\right)$ is more suitable [dC97]. It gives

$$
\begin{aligned}
\operatorname{prob}\left(\bigcup_{\boldsymbol{x} \in X} E_{\boldsymbol{x}}\right) & \geq \sum_{\boldsymbol{x} \in X} \frac{\operatorname{prob}\left(E_{\boldsymbol{x}}\right)^{2}}{\sum_{\boldsymbol{y} \in X} \operatorname{prob}\left(E_{\boldsymbol{x}} \cap E_{\boldsymbol{y}}\right)} \\
& \geq \frac{m q^{2}}{q+(m-1) q^{2}} \\
& \geq \frac{m q^{2}}{q+m q^{2}} \\
& \geq \frac{1}{1+\frac{1}{m q}} \\
& \geq 1-\frac{1}{m q},
\end{aligned}
$$

By taking the maximum of both lower bounds, we obtain our lemma.

\subsection{Estimating the complexity of Algorithm 1}

Here we estimate how many iterations have to be performed in order to break the scheme when no signature is known and when $S=[1 \cdots N]$. For this purpose, we start by lower-bounding the probability that an iteration is successful. Let us bring the following random variables for $i \in\{1,2\}$ :

$$
I_{i}^{\prime} \stackrel{\text { def }}{=} I_{i} \cap J \quad \text { and } \quad W_{i} \stackrel{\text { def }}{=}\left|I_{i}^{\prime}\right| .
$$

By using Lemma 1, we know that an iteration finds a valid signature when there is an $\boldsymbol{x}$ in $\mathscr{C}_{\text {sec }}$ such that

$$
\left|\boldsymbol{x}_{I_{1}^{\prime}}\right|=\left|\boldsymbol{x}_{I_{2}^{\prime}}\right|=p \text {. }
$$


Therefore the probability of success $P_{\text {succ }}$ is lower bounded by

$P_{\text {succ }} \geq \sum_{w_{1}, w_{2}: w_{1}+w_{2} \leqslant n} \operatorname{prob}\left(W_{1}=w_{1}, W_{2}=w_{2}\right) \operatorname{prob}\left\{\exists \boldsymbol{x} \in \mathscr{C}_{\text {sec }}:\left|\boldsymbol{x}_{I_{1}^{\prime}}\right|=\left|\boldsymbol{x}_{I_{2}^{\prime}}\right|=p \mid W_{1}=w_{1}, W_{2}=w_{2}\right\}$

On the other hand, by using Lemma 3 with the set

$$
X \stackrel{\text { def }}{=}\left\{\boldsymbol{x}=\left(x_{j}\right)_{j \in J}:\left|\boldsymbol{x}_{I_{1}^{\prime}}\right|=\left|\boldsymbol{x}_{I_{2}^{\prime}}\right|=p\right\}
$$

which is of size $\left(\begin{array}{c}w_{1} \\ p\end{array}\right)\left(\begin{array}{c}w_{2} \\ p\end{array}\right) 2^{n-w_{1}-w_{2}}$, we obtain

$$
\operatorname{prob}\left\{\exists \boldsymbol{x} \in \mathscr{C}_{\text {sec }}:\left|\boldsymbol{x}_{I_{1}^{\prime}}\right|=\left|\boldsymbol{x}_{I_{2}^{\prime}}\right|=p \mid W_{1}=w_{1}, W_{2}=w_{2}\right\} \geq f(x) .
$$

with

$$
x \stackrel{\text { def }}{=} \frac{\left(\begin{array}{c}
w_{1} \\
p
\end{array}\right)\left(\begin{array}{c}
w_{2} \\
p
\end{array}\right) 2^{n-w_{1}-w_{2}}}{2^{n-k}}=\left(\begin{array}{c}
w_{1} \\
p
\end{array}\right)\left(\begin{array}{c}
w_{2} \\
p
\end{array}\right) 2^{k-w_{1}-w_{2}}
$$

The first quantity is clearly equal to

$$
\operatorname{prob}\left(W_{1}=w_{1}, W_{2}=w_{2}\right)=\frac{\left(\begin{array}{c}
n \\
w_{1}
\end{array}\right)\left(\begin{array}{c}
n-w_{1} \\
w_{2}
\end{array}\right)\left(\begin{array}{c}
N-n \\
(K+k+l) / 2-w_{1}
\end{array}\right)\left(\begin{array}{c}
N-n-(K+k+l) / 2+w_{1} \\
(K+k+l) / 2-w_{2}
\end{array}\right)}{\left(\begin{array}{c}
N \\
(K+k+l) / 2
\end{array}\right)\left(\begin{array}{c}
N-(K+k+l) / 2 \\
(K+k+l) / 2
\end{array}\right)} .
$$

Plugging in the expressions obtained in (22) and (23) in (21) we have an explicit expression of a lower bound on $P_{\text {succ }}$. The number of iterations for our attack to be successful is estimated to be of order $\frac{1}{P_{\text {succ }}}$. We obtain therefore an upper-bound on the expected number of iterations, what we denote by UpperBound. Table 2 shows for various KKS parameters, $p$ and $l$ the expected number of iterations.

Table 2. KKS Parameters with the corresponding value of $\frac{1}{P_{\text {succ }}}$.

\begin{tabular}{l|c|l|l|l|l|l|l|c} 
Article & $\rho$ & $n$ & $l$ & $p$ & $n^{\prime} \stackrel{\text { def }}{=} \mathbb{E}\left\{\left|I^{\prime}\right|\right\}$ & $R$ & $N$ & UpperBound \\
\hline [KKS97] & $\frac{60}{1023} \approx 0.059$ & 1,023 & 8 & 1 & 91 & $\frac{192}{300} \approx 0.064$ & 3,000 & 111.26 \\
& $\frac{60}{1023} \approx 0.059$ & 1,023 & 14 & 2 & 91 & $\frac{192}{3000} \approx 0.064$ & 3,000 & 14.17 \\
\hline$[$ KKS05] & $\frac{48}{255} \approx 0.188$ & 255 & 8 & 1 & 66 & $\frac{260}{1200} \approx 0.208$ & 1,200 & 12.17 \\
& $\frac{48}{255} \approx 0.188$ & 255 & 14 & 2 & 66 & $\frac{260}{1200} \approx 0.208$ & 1,200 & 2.76 \\
\hline [KKS97] & $\frac{48}{180} \approx 0.267$ & 180 & 8 & 1 & 65 & $\frac{335}{1100} \approx 0.305$ & 1,100 & 6.07 \\
& $\frac{48}{180} \approx 0.267$ & 180 & 15 & 2 & 65 & $\frac{335}{1100} \approx 0.305$ & 1,100 & 1.82 \\
\hline [BMJ11] & $1 / 2$ & 320 & 12 & 1 & 165 & $1 / 2$ & 11,626 & 1.24 \\
\hline [BMJ11] & $1 / 2$ & 448 & 13 & 1 & 230 & $1 / 2$ & 16,294 & 1.34 \\
\hline [BMJ11] & $1 / 2$ & 512 & 13 & 1 & 264 & $1 / 2$ & 18,586 & 1.39 \\
\hline [BMJ11] & $1 / 2$ & 768 & 13 & 1 & 395 & $1 / 2$ & 27,994 & 1.61 \\
\hline [BMJ11] & $1 / 2$ & 1,024 & 14 & 1 & 527 & $1 / 2$ & 37,274 & 1.85
\end{tabular}

\subsection{Number of operations of one iteration}

The complexity of one iteration of Algorithm 1 is $C(p, l)=C_{\text {Gauss }}+C_{\text {hash }}+C_{\text {check }}$ where $C_{\text {Gauss }}$ is the complexity of a Gaussian elimination, $C_{\text {hash }}$ is the complexity of hashing all the $\boldsymbol{x}_{1}$ 's and $C_{\text {check }}$ is the complexity of checking all the $\boldsymbol{x}_{2}$ 's with the following expressions:

$$
\begin{aligned}
C_{\text {Gauss }} & =O((N+k)(N-k)(N-k-l))=O\left(N^{3}\right) \\
C_{\text {hash }} & =O\left(\left(\begin{array}{c}
(K+k+l) / 2 \\
p
\end{array}\right)\right) \\
C_{\text {check }} & =O\left(\frac{1}{2^{l}}(N-K-l)^{2}\left(\begin{array}{c}
(K+k+l) / 2 \\
p
\end{array}\right)\right)
\end{aligned}
$$


The last expression giving $C_{\text {check }}$ comes from the fact that the algorithm considers $\left(\begin{array}{c}(K+k+l) / 2 \\ p\end{array}\right)$ elements $\boldsymbol{x}_{2}$, and for each of these candidates, we check about $O\left(\frac{1}{2^{l}}\left(\begin{array}{c}(K+k+l) / 2 \\ p\end{array}\right)\right)$ elements $\boldsymbol{x}_{1}$ 's, which involves a matrix multiplication in Step 4.2. Let us note that $l$ will be chosen such that $C_{\text {hash }}$ and $C_{\text {check }}$ are roughly of the same order, say $2^{l} \approx\left(\begin{array}{c}(K+k+l) / 2 \\ p\end{array}\right)$.

\section{Experimental Results}

The attack described in Section 4 was implemented in Magma [BCP97] in order to validate the analysis developed in Section 5. Table 3 presents the average number of iterations that were necessary to obtain a codeword of weight in the range $\left[t_{1} \cdots t_{2}\right]$. The average is computed with 100 tests for $p=1$ and 10 tests for $p=2$. The values of $t_{1}$ and $t_{2}$ are taken from [KKS97] and [BMJ11]. The algorithm halts whenever it finds a word in the prescribed set. Note that for [BMJ11], we have taken $t_{1}=n / 2-\frac{3}{2} \sqrt{n}$ and $t_{2}=n / 2+\frac{3}{2} \sqrt{n}$ as advocated by the authors. All the codes that we considered during our simulations were randomly chosen. This setting does not completely comply with the recommendations made by the authors for the schemes given in [KKS97]. In one case, it is suggested to use binary BCH codes of length $n=255$ and dimension $k=48$, and in another case a binary code of length $n=180$ and dimension $k=48$ that was constructed by means of 12 random binary equidistant codes of length 15 , dimension 4 and minimum distance 8 . However, we emphasize that these specific constraints are irrelevant because the attack is generic and only requires public data $(\boldsymbol{F}$ and $\boldsymbol{H})$ and aims at forging a valid signature. We can see in Table 3 that the number of iterations are in accordance with the theoretical upper-bound UpperBound on the value of $\frac{1}{P_{\text {succ }}}$ obtained in the previous section, which is an upper bound on the average number of iterations.

Table 3. Average number of iterations of Algorithm 1.

\begin{tabular}{|c|c|c|c|c|c|c|c|c|c|c|}
\hline Article & $\rho$ & $n$ & $l \mid p$ & $n^{\prime} \stackrel{\text { def }}{=} \mathbb{E}\left\{\left|I^{\prime}\right|\right\}$ & $R$ & $N$ & UpperBound & $t_{1}$ & $t_{2}$ & Iterations \\
\hline \multirow[t]{2}{*}{ [KKS97] } & $\frac{60}{1023} \approx 0.059$ & 1,023 & \begin{tabular}{l|l}
8 & 1
\end{tabular} & 91 & $\frac{192}{3000} \approx 0.064$ & 3,000 & 111.26 & 352 & 672 & 111.65 \\
\hline & $\frac{60}{1023} \approx 0.059$ & 1,023 & 142 & 91 & $\frac{192}{3000} \approx 0.064$ & 3,000 & 14.17 & 352 & 672 & 8.67 \\
\hline \multirow[t]{2}{*}{ [KKS05] } & $\frac{48}{255} \approx 0.188$ & 255 & 81 & 66 & $\frac{260}{1200} \approx 0.208$ & 1,200 & 12.17 & 48 & 208 & 22.32 \\
\hline & $\frac{48}{255} \approx 0.188$ & 255 & 142 & 66 & $\frac{260}{1200} \approx 0.208$ & 1,200 & 2.76 & 48 & 208 & 5.67 \\
\hline [BMJ11] & $1 / 2$ & 320 & \begin{tabular}{|l|l|}
12 & 1 \\
\end{tabular} & 165 & $1 / 2$ & 11,626 & 1.24 & 133 & 187 & 1.13 \\
\hline [BMJ11] & $1 / 2$ & 448 & \begin{tabular}{l|l}
13 & 1 \\
\end{tabular} & 230 & $1 / 2$ & 16,294 & 1.34 & 192 & 256 & 1.24 \\
\hline [BMJ11] & $1 / 2$ & 512 & \begin{tabular}{|l|l|}
13 & 1 \\
\end{tabular} & 264 & $1 / 2$ & 18,586 & 1.39 & 222 & 290 & 1.42 \\
\hline [BMJ11] & $1 / 2$ & 768 & \begin{tabular}{|l|l|}
13 & 1 \\
\end{tabular} & 395 & $1 / 2$ & 27,994 & 1.61 & 342 & 426 & 1.83 \\
\hline [BMJ11] & $1 / 2$ & $\mid 1,024$ & \begin{tabular}{|l|l|l}
14 & 1
\end{tabular} & 527 & $1 / 2$ & 37,274 & 1.85 & 464 & 560 & 1.83 \\
\hline
\end{tabular}

\section{Concluding Remarks}

Design principles. As explained in Section 3, the parameters of the KKS scheme were chosen in order to make decoding of $\mathscr{C}_{\text {known }}$ intractable when the weight of errors is in the range $\left[t_{1} \cdots t_{2}\right]$, where $\mathscr{C}_{\text {known }}$ denotes the code defined by the parity-check matrix $\boldsymbol{H}$. In [BMJ11], it is further required that $\mathscr{C}_{\text {known }}$ is of minimum distance greater than $4 n$. Both requirements are clearly insufficient to ensure that the scheme is secure as demonstrated by this paper. We suggest here to replace all these requirements by choosing the parameters such as to make our attack impracticable. This algorithm is exponential in nature when the parameters are well chosen. If we want to avoid that the knowledge of a message/signature pair allows to recover the secret key, this implies for instance that the rate $R$ of $\mathscr{C}_{\text {known }}$ should be significantly larger than $2 \rho$, that is twice the rate of the secret code $\mathscr{C}_{\text {hidden }}$. This would change the parameters of the scheme significantly and give much larger key sizes than has been proposed in [KKS97,KKS05,BMJ11]. 
Relating the security to the problem of decoding a linear code. The attack which has been suggested here is nothing but a well known algorithm for finding low weight codewords or for decoding a generic linear code. It just happens that this algorithm is much more powerful here than for a random linear code due to the peculiar nature of the code it is applied to. However as mentioned above, this attack is exponential in nature and can easily be defeated by choosing the parameters appropriately. It would be interesting to analyze the relationship of the problem of breaking the KKS scheme with decoding problems in more depth, or to prove that the problem which has to be solved is indeed NP hard.

Non-binary codes. Obviously there is a non binary version of the KKS scheme which would deal with codes defined over larger alphabets. The benefits of the generalized scheme are questionable. The attack presented here generalizes easily to higher order fields. What is more, moving to non-binary fields seems to be a poor idea in terms of security. For instance, whereas a message/signature pair reveals only half the positions of $J$ in the binary case, in the $q$-ary case we expect to obtain roughly a fraction $\frac{q-1}{q}$ of positions of $J$, which is significantly larger.

Decoding one out of many. Another approach could have been used for attacking the scheme. Let us denote by $\boldsymbol{s}_{1}, \cdots, \boldsymbol{s}_{k}$ the columns of $\boldsymbol{F}$. These vectors can be considered as $k$ syndromes of codewords of $\mathscr{C}_{\text {hidden }}$ with respect to the parity-check matrix $\boldsymbol{H}$. If we want to obtain one message/pair we can try to find an error $\boldsymbol{e}_{i}$ of weight in the range $\left[t_{1} \cdots t_{2}\right]$ such that $\boldsymbol{H} \boldsymbol{e}_{i}^{T}=\boldsymbol{s}_{i}$. This suggests to use "the decoding one out of many" approach [Sen11], that is we have $k$ words to decode and we want to decode at least one of them. This problem can be solved more efficiently than just decoding one instance. We can even refine this approach by considering all possible syndromes obtained by all possible (non-zero) combinations $\sum_{i} \alpha_{i} s_{i}$. In this case, we would have to solve "a decoding one out of many" problem with $2^{k}-1$ instances. However a naive use of the results of [Sen11] would be far from indicating that all the parameters of [KKS97,KKS05,BMJ11] are easily broken by this approach.

\section{References}

[BCP97] W. Bosma, J. J. Cannon, and Catherine Playoust. The Magma algebra system I: The user language. J. Symb. Comput., 24(3/4):235-265, 1997.

[BF02] A. Barg and G. D. Forney. Random codes: Minimum distances and error exponents. IEEE Transactions on Information Theory, 48(9):2568-2573, September 2002.

[BLP11] D. J. Bernstein, T. Lange, and C. Peters. Smaller decoding exponents: ball-collision decoding. In Proceedings of Crypto 2011, 2011. to appear.

[BMJ11] P. S.L.M. Barreto, R. Misoczki, and M. A. Simplicio Jr. One-time signature scheme from syndrome decoding over generic error-correcting codes. Journal of Systems and Software, 84(2):198 $-204,2011$.

[CFS01] N. T. Courtois, M. Finiasz, and N. Sendrier. How to achieve a McEliece-based digital signature scheme. Lecture Notes in Computer Science, 2248:157-174, 2001.

[Com74] L. Comtet. Advanced Combinatorics. Reidel, Dordrecht, 1974.

[COV07] P.L. Cayrel, A. Otmani, and D. Vergnaud. On Kabatianskii-Krouk-Smeets Signatures. In Proceedings of the first International Workshop on the Arithmetic of Finite Fields (WAIFI 2007), Springer Verlag Lecture Notes, pages 237-251, Madrid, Spain, June 21-22 2007.

[dC97] D. de Caen. A lower bound on the probability of a union. Discrete Mathematics, 169:217-220, 1997.

[Dum91] I. Dumer. On minimum distance decoding of linear codes. In Proc. 5th Joint Soviet-Swedish Int. Workshop Inform. Theory, pages 50-52, Moscow, 1991.

[Dum96] I. Dumer. Suboptimal decoding of linear codes : partition techniques. IEEE Transactions on Information Theory, 42(6):1971-1986, 1996.

$\left[\mathrm{FGO}^{+} 10\right]$ Jean-Charles Faugère, Valérie Gauthier, Ayoub Otmani, Ludovic Perret, and Jean-Pierre Tillich. A distinguisher for high rate McEliece cryptosystems. Cryptology ePrint Archive, Report 2010/331, 2010. http://eprint.iacr.org/.

[FS09] M. Finiasz and N. Sendrier. Security bounds for the design of code-based cryptosystems. In M. Matsui, editor, Asiacrypt 2009, volume 5912 of LNCS, pages 88-105. Springer, 2009. 
[KKS97] G. Kabatianskii, E. Krouk, and B. Smeets. A digital signature scheme based on random errorcorrecting codes. In Proceedings of the 6th IMA International Conference on Cryptography and Coding, pages 161-167, London, UK, 1997. Springer-Verlag.

[KKS05] G. Kabatiansky, E. Krouk, and S. Semenov. Error Correcting Coding and Security for Data Networks: Analysis of the Superchannel Concept. John Wiley \& Sons, 2005.

[MS86] F. J. MacWilliams and N. J. A. Sloane. The Theory of Error-Correcting Codes. North-Holland, Amsterdam, fifth edition, 1986.

[Sen11] N. Sendrier. Decoding one out of many, 2011. preprint.

[Ste88] J. Stern. A method for finding codewords of small weight. In G. D. Cohen and J. Wolfmann, editors, Coding Theory and Applications, volume 388 of Lecture Notes in Computer Science, pages 106-113. Springer, 1988. 